\title{
SIMULATION BASED PERFORMANCE OF MUMBAI-PUNE EXPRESSWAY SCENARIO FOR VEHICLE-TO-VEHICLE COMMUNICATION USING IEEE 802.11P
}

\author{
Vaishali D. Khairnar ${ }^{1}$, Ketan Kotecha ${ }^{2}$ \\ ${ }^{1}$ Research Scholar Institute of Technology Nirma University Ahmadabad \\ E-mail: khairnar.vaishali3@gmail.com \\ ${ }^{2}$ Director Institute of Technology Nirma University Ahmadabad \\ E-mail: director.it@nimauni.ac.in
}

\begin{abstract}
Traffic safety applications using vehicle-to-vehicle (V2V) communication is an emerging technology and promising area within the ITS environment. Many of these applications require real-time communication with high reliability; to meet a real-time deadline, timely and predictable access to the channel. The medium access method used in 802.11p, CSMA with collision avoidance, does not guarantee channel access before a finite deadline. The well-known property of CSMA is undesirable for critical communications scenarios. The simulation results reveal that a specific vehicle is forced to drop over $80 \%$ of its packets because no channel access was possible before the next message was generated. To overcome this problem, we propose to use STDMA for realtime data traffic between vehicles. The real-time properties of STDMA are investigated by means of the highway road simulation scenario, with promising results.
\end{abstract}

Keywords: CSMA, STDMA, V2V, VANET

\section{Introduction}

The new emerging applications for traffic safety found within the vehicular ad-hoc network environments which can be classified as real-time system. Existing vehicle-to-vehicle safety systems together with new cooperative systems using wireless data communication between vehicles which can potentially decrease the number of accidents on the highway road in India, i.e., transmit the messages within deadline time. In addition, requirements on high reliability and low delay are imposed on wireless communication system [1]. For example, Lane departure warning messages merge assistance and emergency vehicle routing are all examples of applications [2]. Information that is delivered correctly, but after the deadline in a real-time communication system, is not only useless, but can also have severe consequences for the traffic safety system. This problem is pointed out in [3-5]. In most cases, the extremely low delays required by traffic safety applications, the need for ad-hoc network architectures support direct vehicle-to-vehicle communication. The original IEEE 802.11, intended for WLAN, has two drawbacks within its MAC technique CSMA; it can cause unbounded delays before channel access as well as collisions on the channel. The MAC protocol decides who has right data/packet to transmit next on the shared communication channel. In CSMA, the node first listens to the channel an if the channel is free for certain amount of time period, then the node transmits data/packets directly with the implication that another node can have conducted the exact same procedure, resulting in a collision on the channel. CSMA is used by IEEE 802.11 family as well as its wired counterpart IEEE 802.3 Ethernet. One of the reasons for the success of both WLAN and Ethernet is the straightforward implementation of the standard resulting in reasonable priced equipment.

\section{Medium Access Control}

Vehicular ad-hoc network (VANETs) is a spontaneous, unstructured network based on direct vehicle-to-vehicle $(\mathrm{V} 2 \mathrm{~V})$ communication and its topology is changing constantly due to high mobility of vehicle nodes on highway road. In VANET it is harder to deploy a MAC scheme that is relying on a centralized controller.

\subsection{IEEE 802.11p/DSRC Protocol}

The IEEE $802.11 \mathrm{p}$ standard (WAVE) emerges from the allocation of the Dedicated Short Range Communications (DSRC) spectrum band in the United States and the work done to define the technology 
to be used in this band. There are two types of channels in DSRC, all of them with a $10 \mathrm{MHz}$ width: the control channel $(\mathrm{CCH})$ and the service channel $(\mathrm{SCH})$. The $\mathrm{CCH}$ is restricted to safety communications only and the SCHs are available both for safety and non-safety use. Applications for vehicular communications can be placed in three main categories - traffic safety, traffic efficiency and value-added services (e.g. infotainment/business) [7-11].

In Europe, the spectrum allocated by the ETSI for cooperative safety communications has a range $5,875-5,925 \mathrm{GHz}$. It is divided into traffic safety $(30 \mathrm{MHz})$ and traffic efficiency $(20 \mathrm{MHz})$. In the traffic safety spectrum, two SCHs and one $\mathrm{CCH}$ are allocated. In the traffic efficiency two SCHs are allocated [11]. WAVE is fully intended to serve as an international standard, which is meant to: describe the functions and services required by WAVE stations to operate in VANETs, and define the WAVE signalling technique and interface functions that are controlled by the IEEE 802.11 MAC. WAVE is an amendment to the Wireless Fidelity (Wi-Fi) standard IEEE 802.11 [18,12].

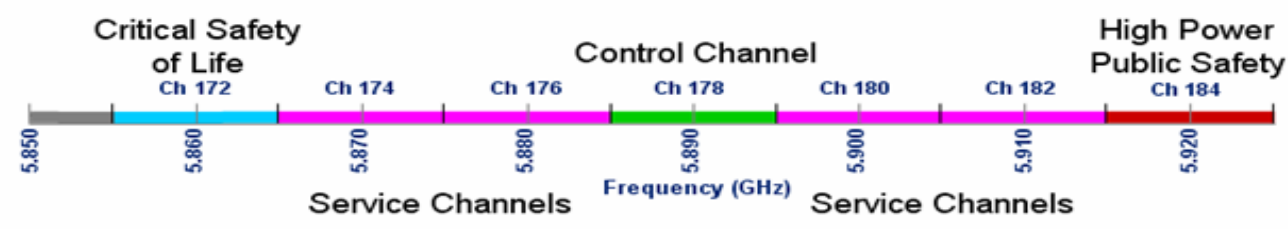

Figure 1. DSRC spectrum band and channels

\subsection{MAC Layer}

For the adaptation of IEEE 802.11a to IEEE 802.11p, no changes in the MAC layer have been done $[20-22]$.

\subsubsection{Overview of MAC services}

\subsubsection{Data service}

This service provides peer entities in the LLC (Local Link Control) MAC sub-layer with the ability of exchanging MSDUs (MAC Service Data Units) using the underlying PHY-layer services. This delivery of MSDUs is performed in an asynchronous way, on a connectionless basis [15].

\subsubsection{MSDU ordering}

In nQSTAs, the ones simulated in this paper, there are two service classes within the data service. By selecting the desired service class, each LLC entity initiating the transfer of MSDUs is able to control whether MAC entities are or are not allowed to reorder those MSDUs at reception [25-27].

\subsection{MAC sub-layer functional description}

\subsubsection{MAC architecture}

The MAC architecture can be described as shown on Figure 3 as providing the Point Coordination Function (PCF) and Hybrid Coordination Function (HCF) through the services of the Distributed Coordination Function (DCF) [23, 24].

\subsubsection{DCF}

DCF is the fundamental MAC technique in the IEEE 802.11 standard. It employs an access function performed by the CSMA/CA algorithm and a collision management function carried out by the binary exponential back-off procedure.

\subsubsection{P C F}

The original IEEE 802.11 standard defines another coordination function in the MAC layer. It is only available in structure mode networks, where the nodes are interconnected through at least one AP in the network. In the scope of this paper, PCF is not used.

\subsubsection{H C F}

$\mathrm{HCF}$ is a coordination function that enables the QoS facility. It is only usable in networks that make use of QoS, so it is only implemented in the QSTAs. The HCF combines functions from the DCF and PCF with some enhanced, QoS-specific mechanisms and frame subtypes to allow a uniform set of 
frame exchange sequences to be used for QoS data transfers. The HCF uses both a controlled channel access mechanism, HCCA, for contention-free transfer and a contention-based channel access method mechanism, EDCA.

\subsubsection{H C C A}

HCCA works similarly to PCF. It uses a QoS-aware centralized coordinator, called a Hybrid Coordinator $(\mathrm{HC})$, and operates under rules that are different from the $\mathrm{PC}$ of the PCF.

\subsection{Self-Organizing Time Division Multiple Access (STDMA) MAC Layer Algorithm}

The STDMA algorithm, invented in [16, 17], is already used in commercial applications for surveillance, i.e., the Automatic Identification System (AIS) used by ships and the VHF data link (VDL) mode 4 system used by the avionics industry. By adding data communication based on STDMA, more reliable information can be obtained about other ships and airplanes in the vicinity and thereby accidents can be avoided. STDMA is a decentralized MAC scheme where the network members themselves are responsible for sharing the communication channel. Nodes utilizing this algorithm, will broadcast periodic data messages containing information about their position. The algorithm relies on the nodes being equipped with GPS receivers. Time is divided into frames as in a TDMA system and all stations are striving for a common frame start. These frames are further divided into slots, which typically corresponds to one packet duration. The frame of AIS and VDL model 4 is one minute long and is divided into 2250 slots of approximately $26 \mathrm{~ms}$ each. All network members start by determining a report rate. Then follows four different phases; initialisation, network entry, first frame, and continuous operation. During the initialisation, a node will listen to the channel activity during one frame length to determine the slot assignments. In the network entry phase, the node determines its own transmission slots within each frame according to the following rules: (i) calculate a nominal increment (NI) by dividing the number of slots with the report rate, (ii) randomly select a nominal start slot (NSS) drawn from the current slot up to NI, (iii) determine a selection interval (SI) of slots as $20 \%$ of NI and put this around the NSS according to Figure. 4, (iv) now the first actual transmission slot is determined by picking a slot randomly within SI and this will be the nominal transmission slot (NTS). If the chosen NTS is occupied, then the closest free slot within SI is chosen. When the first NTS is reached in the super frame, the node will enter the third phase called the first frame.

\begin{tabular}{|c|c|c|c|c|}
\hline \multirow{2}{*}{$\begin{array}{c}\text { Application layer } \\
\text { Message sub-layer }\end{array}$} & \multicolumn{2}{|c|}{ Safety Appl. } & \multirow{2}{*}{$\begin{array}{l}\text { Non-Safety } \\
\text { Applications }\end{array}$} & \multirow{2}{*}{ Application layer } \\
\hline & \multirow{3}{*}{ 预 } & $J 2735$ & & \\
\hline \multirow{2}{*}{$\begin{array}{l}\text { Network and } \\
\text { transport layers }\end{array}$} & & WSMP & TCP/UDP & Transport layer \\
\hline & & 1609.3 & IPv6 & Network layer \\
\hline LLC sub-layer & \multicolumn{3}{|c|}{ IEEE 802.2} & \multirow{3}{*}{ Data link layer } \\
\hline MAC sub-layer ext. & \multicolumn{3}{|c|}{ WAVE 1609.4} & \\
\hline MAC sub-layer & \multicolumn{3}{|c|}{ IEEE 802.11} & \\
\hline Physical layer & \multicolumn{3}{|c|}{ IEEE 802.11} & Physical layer \\
\hline
\end{tabular}

Figure 2. An overview of the WAVE protocol stack containing both a road traffic safety applications' part as well as a nonsafety part

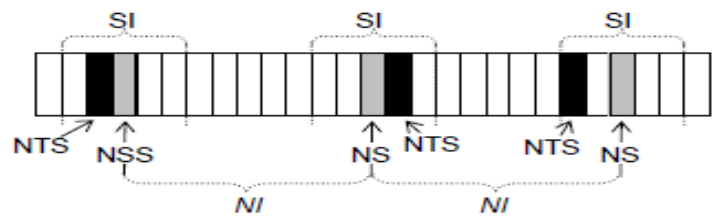

Figure 4. The STDMA algorithm in the first frame phase

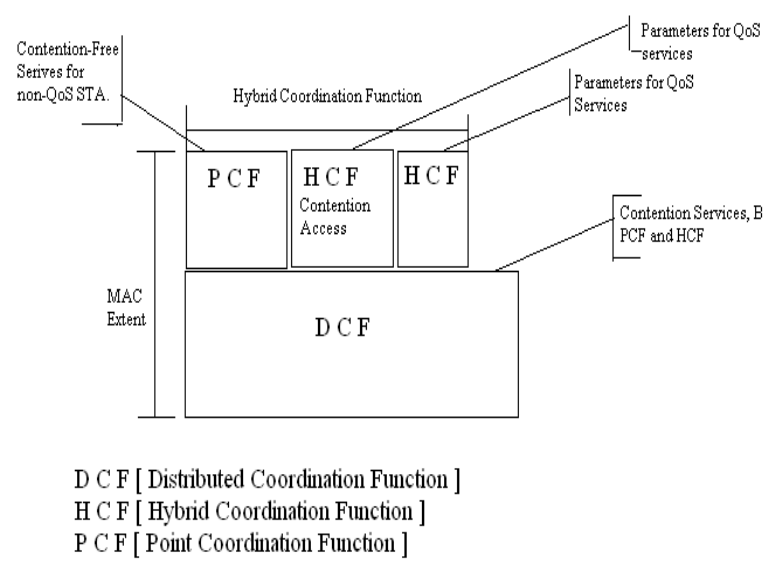

Figure 3. MAC architecture

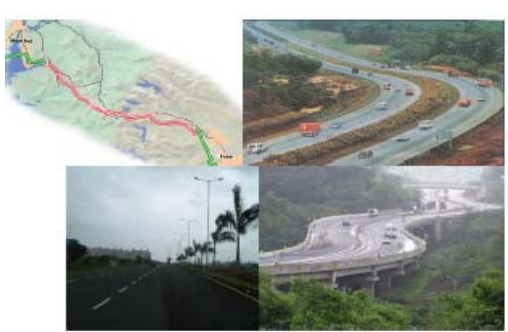

Figure 5. Scenario of Mumbai-Pune Highway Road 
After the first frame phase (which lasts for one frame) when all NTS were decided, the station will enter the continuous operation phase, using the NTSs decided during the first frame phase for transmission. During the first frame phase, the node draws a random integer $n \in\{3, \ldots, 8\}$ for each NTS. After the NTS has been used for $n$ frames, a new NTS will be allocated in the same SI as the original NTS.

\section{Simulations}

The real-time properties of the system, the interesting issue here is how the two MAC protocols will influence the capability of each sending vehicle node to timely deliver data/messages packets. We are dealing with an uncontrolled network since the number of network vehicle nodes cannot be determined in advance as we are considering vehicles are controlled by humans. On the highway road, the highest relative speeds are found and this causes the network topology to change often and more rapidly. If a traffic accident occurs, many vehicles could be gathered in a small geographic area implying troubles with access to the shared wireless communication channel for individual vehicle nodes. The promising emerging application within VANET is a cooperative awareness system such as the automatic identification system for the ships, where the vehicles will exchange location messages with each other to build up a map of its surrounding and use this for different traffic safety efficiency application [27]. Consequently, we have also chosen to use broadcasted, time-driven location messages as the data traffic model in the simulator. Many traffic safety systems will rely on vehicles periodically broadcasting messages containing their current state. We have developed a simulator using Open Street Map, eWorld, SUMO version 0.12 .3 (traffic simulator), NS-2 version 2.34 (Network Simulator) and TraNs version 1.2 (Intermediate simulator between SUMO and NS2) also we require Gnu plot to plot the graphics presentation (Figure 6 for Simulation Flow diagram) where each vehicle sends a location message according to a predetermined range of 5 or $10 \mathrm{~Hz}$. The vehicle traffic scenario is a Mumbai-Pune Highway Road of 120 kilometres (km), i.e., 12000 meter with 3 lanes in each direction (i.e., total 6 lanes including both the directions), see Figure 5.

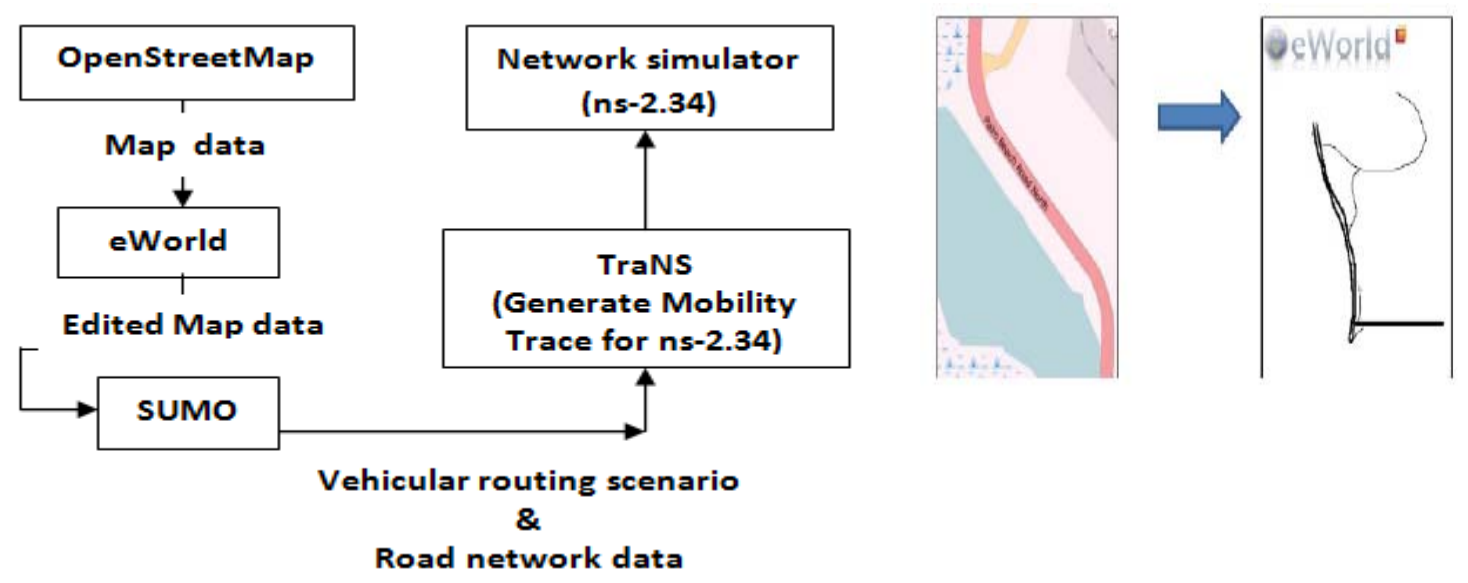

Figure 6. Simulation Flow Diagram

The Mumbai-Pune Highway Road scenario is chosen because here the highest relative speeds (i.e., $\min 80 \mathrm{~km} / \mathrm{h}$ to $\max 120$ or above $\mathrm{km} / \mathrm{h}$ ) in vehicular environments are found and hence it should constitute the biggest challenge for the MAC layer. The vehicles are entering each lane of the highway road according to a Poisson process with a mean inter-arrival time of 3 seconds. The channel model is a simple circular transmission model where all vehicles within a certain sensing range will sense and receive packets perfectly. The simulated sensing ranges are $500 \mathrm{~m}$ and $1000 \mathrm{~m}$. We have tried to focus on how the two MAC methods perform in terms of time between channel access requests until actual channel access within each vehicle node [28]. The transfer rate is chosen to be the lowest rate supported by $802.11 \mathrm{p}$, namely $3 \mathrm{Mbps}$ as in Table 1 . 
Table 1. Simulation parameter setting for Mumbai-Pune Highway Road scenario simulation

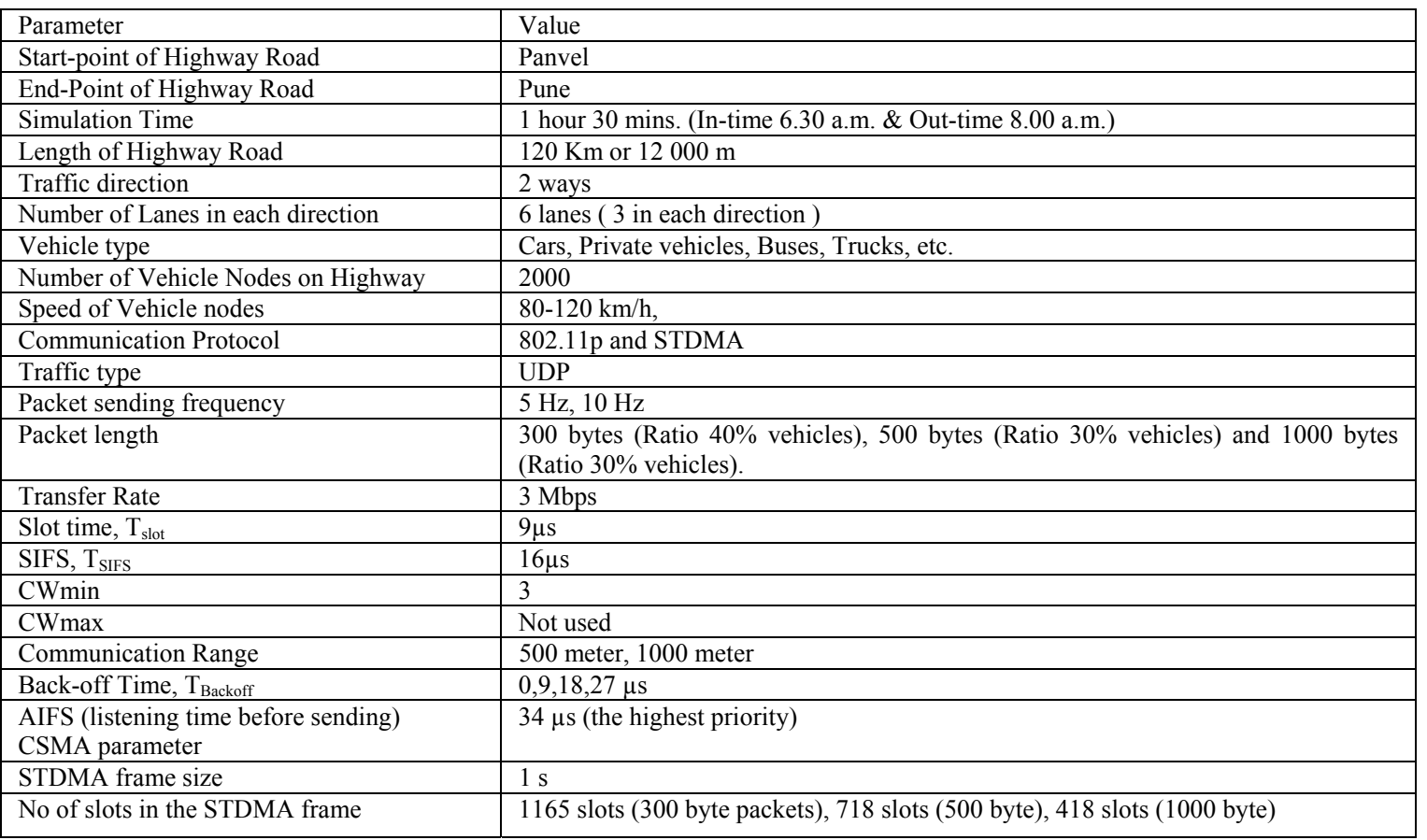

The channel model is a simple circular sensing range model, Figure 7, in which every vehicle node within the sensing area receives the message perfectly. Note the vehicle nodes could be exposed to two concurrent transmissions, where transmitters TX1 and TX2 are sending at the same time since the transmitters cannot hear each other: the receivers RX1, RX2, and RX3 on Figure 7 will then experience collisions of the two ongoing transmissions, unless some sort of power control or multi-user detection is used. The simulation has been carried out with three different packet lengths: $\mathrm{N}=300,500$ and 1000 bytes and two different sensing ranges 500 and 1000 meters. We used in our CSMA simulations, all vehicles use the MAC method of 802.11p, and hence each vehicle must listen before sending and back-off if the channel is busy or becomes busy during the automatic identification system. A broadcast packet will experience at most one back-off procedure due to the lack of ACKs in a broadcast system. Since all data traffic in our simulation scenario has the same priority, only the highest priority automatic identification system and $\mathrm{CW}_{\min }$ have been used and therefore all transmitters will have the same $\mathrm{T}_{\mathrm{AIFS}}$ value $(34 \mu \mathrm{s})$. The back-off time is the product of the slot time, $\mathrm{T}_{\text {slot }}$, and a random integer uniformly distributed in the interval [0,3] implying four possible back-off times, $T_{\text {backoff: }} 0,9,18$ and $27 \mu$ s respectively. The STDMA algorithm found in automatic identification system cannot be used right away since the dynamics of a vehicular ad-hoc network and a shipping network are quite different. There is a natural inertia inherent in a shipping system that is not present in the vehicular environment.

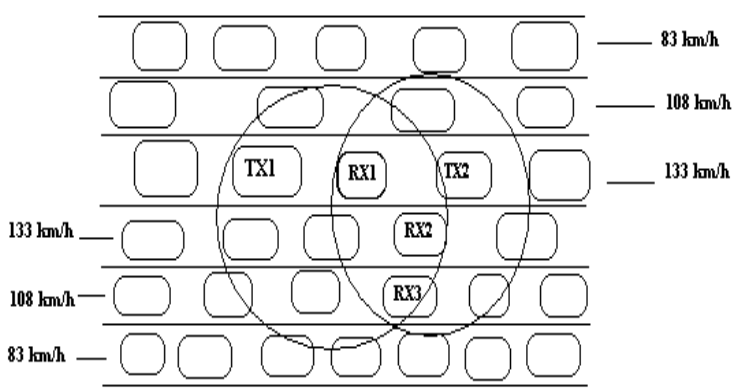

Figure 7. Simulation Set-up

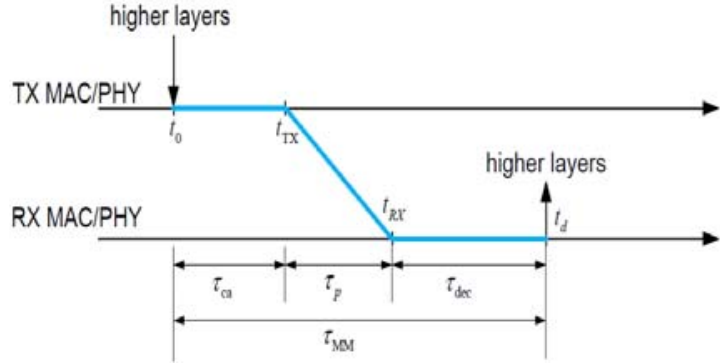

Figure 8. Mac-to-Mac (E2E) Delay

Both MAC protocols used in the simulation are assumed to use the same physical layer from $802.11 \mathrm{p}$. The frame duration, $\mathrm{T}_{\text {frame, }}$, in our simulated STDMA scheme has been set to 1 second and the number of slots is changed inside the frame to cater for different packet lengths. A transfer rate, R of 3 
Mbps has been used and this rate is available with the PHY layer of $802.11 \mathrm{p}$, which has support for eight transfer rates in total where $3 \mathrm{Mbps}$ is the lowest. In the STDMA simulations, the vehicles will go through three phases: initialisation, network entry and first frame, before it ends up in the continuous operations. The vehicle stays in the continuous phase after it has been through the other three. STDMA always guarantees channel access even when all slots are occupied within an SI, in which case a slot belonging to the vehicle node located furthest away will be selected. The time parameters involved in the simulation are selected from the PHY specification of $802.11 \mathrm{p}$. The CSMA transmission time, $\mathrm{T}_{\text {CSMA }}$, consists of an AIFS period $\mathrm{T}_{\mathrm{AIFS}}$ in $34 \mu \mathrm{s}$, a $20 \mu \mathrm{s}$ preamble, $\mathrm{T}_{\text {preamble, and the actual data packet }}$ transmission, $\mathrm{T}_{\text {packet }}$. The STDMA transmission time, $\mathrm{T}_{\text {STDMA }}$, which is the same as the slot time, consists of two guard times, $\mathrm{T}_{\mathrm{GT}}$, of $3 \mu \mathrm{s}$ each, $\mathrm{T}_{\text {preamble, }} \mathrm{T}_{\text {packet, }}$ and two SIFS periods, $\mathrm{T}_{\mathrm{SIFS}}$ of $16 \mu \mathrm{s}$ each derived from the PHY layer in use. The total transmission time for CSMA is

$$
\mathrm{T}_{\mathrm{CSMA}}=\mathrm{T}_{\text {AIFS }}+\mathrm{T}_{\text {preamble }}+\mathrm{T}_{\text {packet }}
$$

and the total transmission time for STDMA is

$$
\mathrm{T}_{\mathrm{STDMA}}=2 \mathrm{~T}_{\mathrm{GT}}+2 \mathrm{~T}_{\mathrm{SIFS}}+\mathrm{T}_{\text {preamble }}+\mathrm{T}_{\text {packet }}
$$

We have assumed that all the vehicle nodes in the system are perfectly synchronized with each other in both MAC protocol scenarios and that in the STDMA case they are also aware of when the frame starts and how many time slots it contains. The delay that takes to a packet sent from the transmitting vehicle until it is decoded by the receiving vehicle at the MAC layer level. This delay is expressed as:

$$
\mathrm{T}_{\mathrm{MM}}=\mathrm{Tca}+\mathrm{Tp}+\mathrm{Tdec}
$$

At the receiver side, to be a packet candidate to be decoded and sent to higher layers, it should have arrived within $100 \mathrm{~ms}$, which is the maximum allowed delay at the receiver vehicle for CAM messages to be considered.

\section{Performance Evaluations of CSMA and STDMA}

Since CSMA will be the prevailing MAC method of emerging standards for VANET based road traffic safety applications. In addition, STDMA as described will also be evaluated, since this MAC method has the potential to fulfil the requirements imposed by VANET based road traffic safety applications. The channel access delay highlights the ability of the MAC method to provide a predictable delay which is a functional requirement. The packet reception probability is a non-functional requirement, i.e., a quality measure determining how well the MAC method schedules transmissions in time and space.

The performance of CSMA and STDMA has been evaluated by means of computer simulations in SUMO and NS2.34. The highway scenario was selected to model the vehicle traffic pattern since the highest relative speeds are found here and therefore it is likely the most stressing case for the MAC methods since stations can show up and quickly disappear again due to high velocities.

A $120 \mathrm{~km}$ highway scenario with 6 lanes, three in each direction, has been used for the simulations. The vehicles arrive at the highway entrance in each direction in each lane according to a Poisson distribution with mean inter-arrival time of three seconds. The vehicle speeds are drawn independently from a Gaussian distribution with a common standard deviation of $1 \mathrm{~m} / \mathrm{s}$, but with three different mean values $(23 \mathrm{~m} / \mathrm{s}, 30 \mathrm{~m} / \mathrm{s}$, and $37 \mathrm{~m} / \mathrm{s})$ depending on lane. The vehicles maintain the same speed as long as they are on the highway and overtaking is not considered (i.e., vehicles may pass in the same lane by driving over each other). The resulting vehicle density is then approximately 120 vehicles/km of highway (in total about 1200 to 2000 vehicles on the highway at the same time). All vehicles are moved every $100 \mathrm{~ms}$. Simulation is carried out by parameters setting in Table 1. Data from the simulations have been collected only when the Mumbai-Pune Highway Road was filled with vehicles. The results from all 20 simulated scenarios using CSMA are shown in Table 2 where the numbers represent the data packet drops in percent. A data packet is dropped or discarded by the vehicle node

\begin{tabular}{|c|c|c|c|c|c|}
\hline \multirow{2}{*}{\multicolumn{2}{|c|}{ CSMA }} & \multicolumn{4}{|c|}{ Sensing range } \\
\hline & & \multicolumn{2}{|c|}{500 meter } & \multicolumn{2}{|c|}{1000 meter } \\
\hline \multicolumn{2}{|c|}{ Data Packet Rate } & $5 \mathrm{~Hz}$ & $10 \mathrm{~Hz}$ & $5 \mathrm{~Hz}$ & $10 \mathrm{~Hz}$ \\
\hline \multirow[t]{2}{*}{ Packet length } & 300 byte & $0 \%$ & $0 \%$ & $0 \%$ & $36 \%$ \\
\hline & 1000 byte & $0 \%$ & $30 \%$ & $45 \%$ & $60 \%$ \\
\hline
\end{tabular}
when the next data packet is generated.

Table 2. Packet drops on average for different data traffic scenarios 
From Table 2 it can be seen that, if 1000 byte long data packets are sent every $100 \mathrm{~ms}$ and the sensing range is 1000 meters, only $45 \%$ of the channel access request will result in actual channel access for $802.11 \mathrm{p}$. But, this value is averaged over all transmissions made by all vehicles in the system which means that certain nodes experience an even worse situation. On Figure 9, the best and worst performance experienced by a single user is depicted together with the average for all users in the system. In the worst case, a vehicle node achieves successful channel access only $14 \%$ of the time, i.e., $85 \%$ of all generated packets in this vehicle node are dropped. When the sensing range is 1000 meters, a vehicle node will complete for the channel with approximately 230 other vehicle nodes.

\subsection{Channel access delay}

On Figure 9, the CDF of the channel access delay for CSMA is depicted for all update rates when using the Nakagami channel model described above. On Figure 9 (a) update rates of 2-6 Hz is shown and as can be seen, no station experiences a channel access delay that is longer than 3 ms. On Figure 9(b), depicting update rates $8-20 \mathrm{~Hz}$, a maximum channel access delay of $12 \mathrm{~ms}$ is encountered for the highest update rate, $20 \mathrm{~Hz}$. We can conclude that with an update rate of $2 \mathrm{~Hz}, 85 \%$ of all generated packets achieve channel access after the mandatory minimum waiting time of an AIFS of $71 \mu \mathrm{s}$, whereas with an update rate of $20 \mathrm{~Hz}$, less than $10 \%$ of all generated packets experience the same minimum wait, implying that $90 \%$ of all initial transmission attempts result in a back-off procedure. In STDMA, the channel access delay is upper-bounded, i.e., a station always knows when it is allowed to transmit during its SI intervals. However, the size of the SI depends on the number of packets transmitted during one second. As the update rate increases, the SI will shrink and thereby the number of slots contained in the SI also reduces. On Figure 10 , the channel access delay for STDMA is depicted with the same update rates and channel model as for CSMA on Figure 9. As can be deduced from Figure 10(a), the worst case channel access delay that STDMA can exhibit is $100 \mathrm{~ms}$ and this occurs when the update rate is set to $2 \mathrm{~Hz}$ (implying $500 \mathrm{~ms}$ between every generated packet). However, $50 \%$ of the generated packets have been transmitted after $50 \mathrm{~ms}$ even in this case. Conversely, the shortest channel access delay occurs for $20 \mathrm{~Hz}$ (i.e., $50 \mathrm{~ms}$ between every generated packet), yielding a maximum channel access delay of $10 \mathrm{~ms}$. The staircase appearance of the curves is due to the number of slots in each SI. It should be noted that the channel access delay encountered in STDMA is neither affected by the channel model nor the network load. Consequently, the same channel access delays are also found for the LOS/OLOS model when STDMA is used. For CSMA using the LOS/OLOS channel model, the channel access delay is depicted on Figure 11. On Figure 12, the Nakagami and LOS/OLOS models are compared for CSMA. It can be concluded that the channel access delay is affected by the channel model in use and that the LOS/OLOS model implies longer channel access delays. This is due to the fact that the LOS/OLOS channel model has a successful packet reception range that is slightly longer than in the Nakagami case, implying that each station has slightly more stations within its radio range. These additional stations keep the channel occupied more often, forcing more stations into the back-off procedure and thereby increasing the channel access delay. When evaluating the channel access delay for CSMA and STDMA it can be concluded that while the minimum delay is smaller for CSMA than for STDMA, the worst case delay is random for CSMA. For STDMA, the worst case channel access delay is known and independent on network load and channel type.

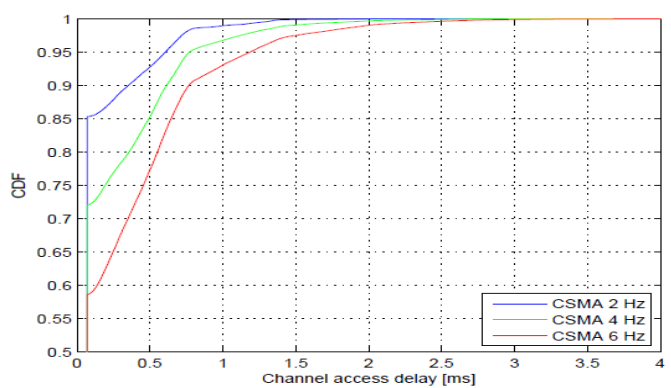

(a)

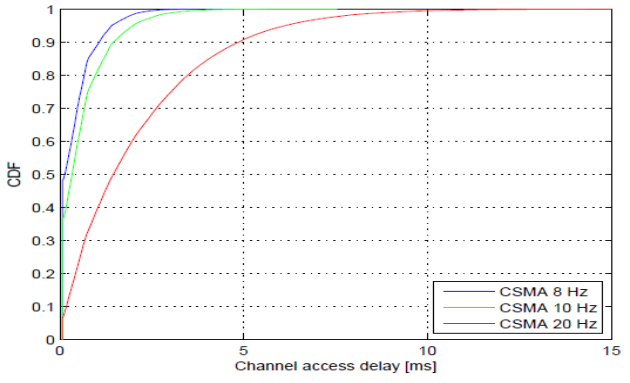

(b)

Figure 9. Channel access delay for CSMA when using the Nakagami model; (a) update rate of 2/4/6 Hz, and (b) update rate of $8 / 10 / 20 \mathrm{~Hz}$

Figure 13(a)-(b) shows the packet reception probability for CSMA and STDMA, respectively, with the update rates: $2 / 4 / 6 / 8 / 10 / 20 \mathrm{~Hz}$ and the Nakagami channel model. The blue upper bound curve, denoted "Genie" on Figures 13-16, is the single transmitter case, i.e., no MAC method is needed as there is only one transmitter in the system and no interferers, implying that this is an unattainable upper bound 
for any network with more than one transmitter. Note that the update rate does not affect the packet reception probability per se, but since more transmissions take place, the probability of interferers is higher, which affects the probability of successful reception. From Figure 13, it can be concluded that STDMA has a higher packet reception probability for all considered rates, i.e., closer to the "Genie" compared to CSMA.

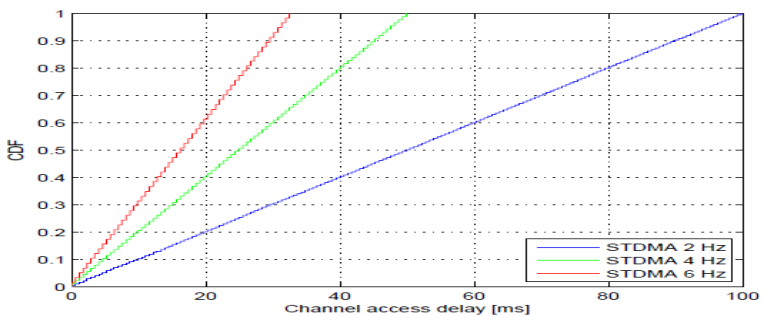

(a)

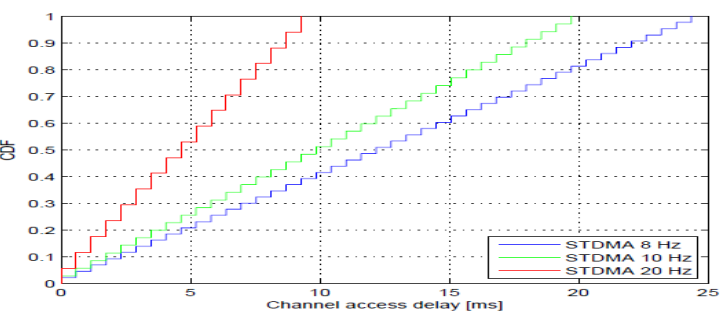

(b)

Figure 10. Channel access delay for STDMA; (a) update rate of 2/4/6 Hz, and (b) update rate of 8/10/20 Hz

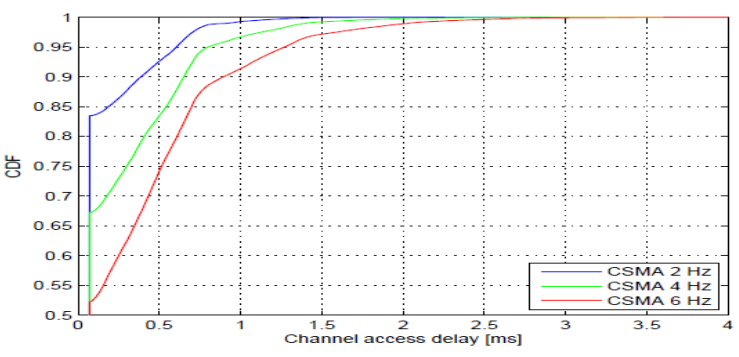

(a)

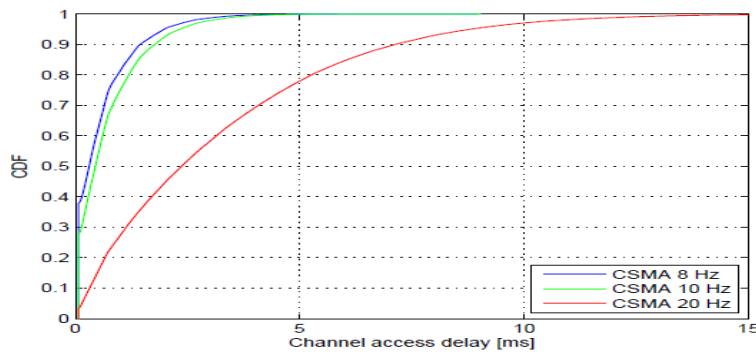

(b)

Figure 11. Channel access delay for CSMA when using the LOS/OLOS model for the following update rates;

(a) $2 / 4 / 6 \mathrm{~Hz}$, and (b) $8 / 10 / 20 \mathrm{~Hz}$

On Figure 14 the two MAC schemes are shown together for all considered rates. When RX is close to TX $(<100$ meters) both MAC methods perform equally well. However, when the TX-RX distance increases, STDMA achieves a higher packet reception probability. At a TX-RX distance of 300 meters and an update rate of $6 \mathrm{~Hz}$ (Figure 14 (c)) and $8 \mathrm{~Hz}$ (Figure 14(d)), there is roughly a 20\% performance gain with STDMA as compared to CSMA. For an update rate of $20 \mathrm{~Hz}$, which can be regarded as an overloaded scenario, there is too much interference in the system for any of the two protocols, and the gap to the "genie" is considerable. In CSMA, the overloaded scenario causes stations within radio range to transmit simultaneously resulting in decoding failures at the receivers. The simultaneous transmissions occur since many stations are forced into back-off, and their back-off counters run the risk of reaching zero at the same time. For STDMA, the overloaded scenario implies that many slots are used by more than one station, resulting in a higher probability of decoding errors at the receivers and yet these slots are perceived as busy due to signal strengths above the CSth. Thereby stations are sometimes forced to select a slot within its SI that is perceived as busy but with missing position information, i.e., the protocol cannot take advantage of its ability to schedule transmissions in space.

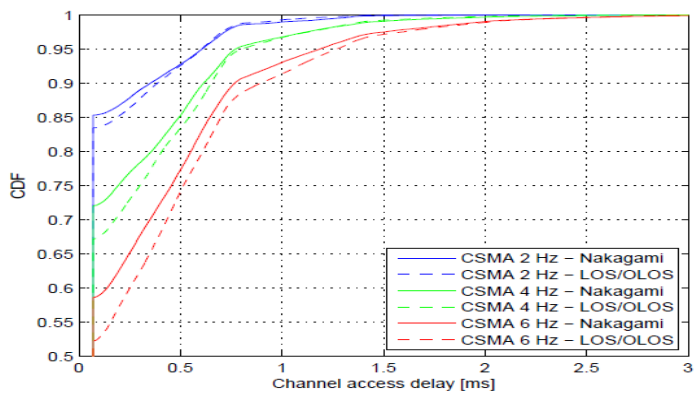

(a)

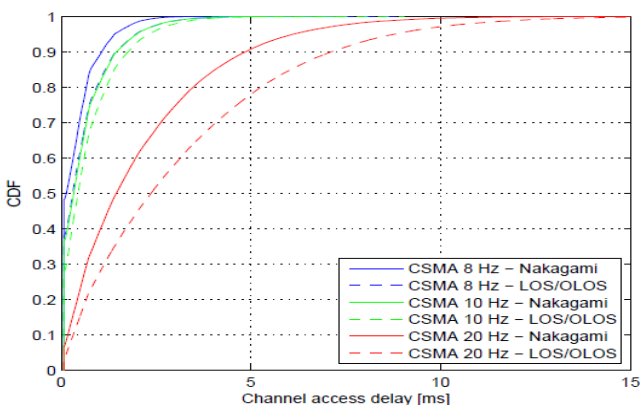

Figure 12. Channel access delay for CSMA when using the LOS/OLOS model and the Nakagami model for the following update rates; (a) $2 / 4 / 6 \mathrm{~Hz}$, and (b) $8 / 10 / 20 \mathrm{~Hz}$ 


\subsection{Packet reception probability}
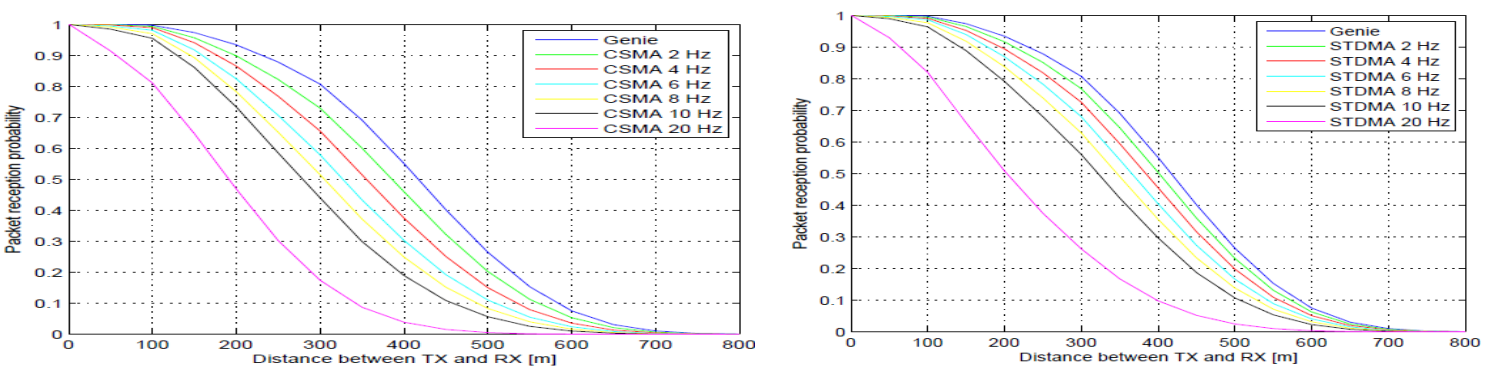

(a)
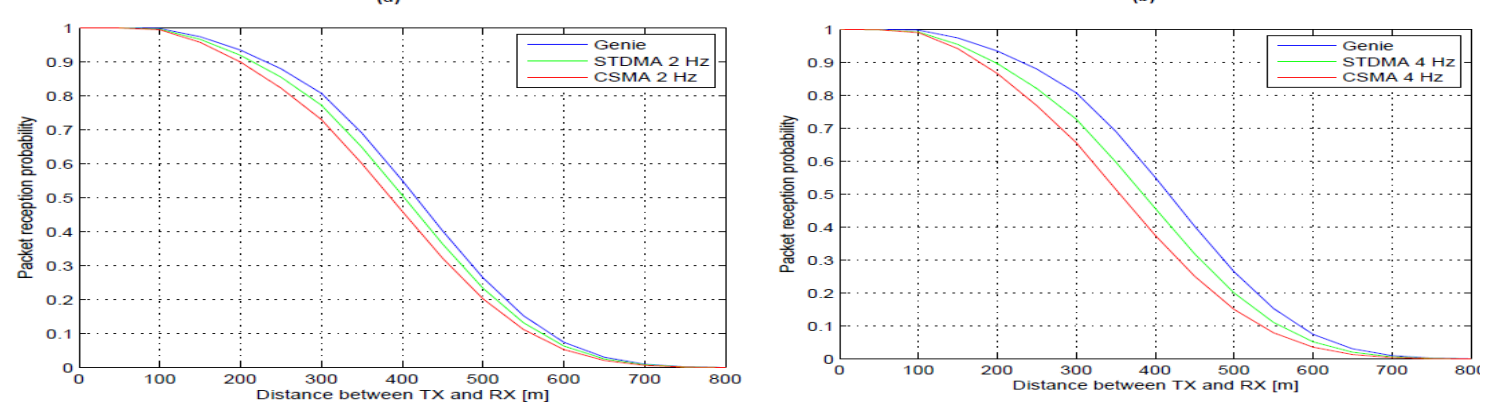

(a) Update rate $2 \mathrm{~Hz}$

(b) Update rate $4 \mathrm{~Hz}$

Figure 13. Packet reception probability for update rates of 2,4,6,8,10,16,20 Hz using the Nakagami model;

(a) CSMA, and (b) STDMA

On Figure 15 the packet reception probability for CSMA and STDMA when using the LOS/OLOS model is depicted. It can be seen that the LOS/OLOS model has about 400 meters longer communication range than the Nakagami model, i.e., the packet reception probability approaches 0 for receivers approximately 400 meters further away.

On Figure 16 a comparison between CSMA and STDMA for different update rates is shown when using the LOS/OLOS model. The results show that STDMA performs better than CSMA for all settings also for this channel model. At a TX-RX distance of 300 meters, on Figure 14(c) update rate of $6 \mathrm{~Hz}$ and on Figure 14(d) update rate of $8 \mathrm{~Hz}$, STDMA has almost a $20 \%$ better performance than CSMA. Consequently, STDMA is more reliable than CSMA.
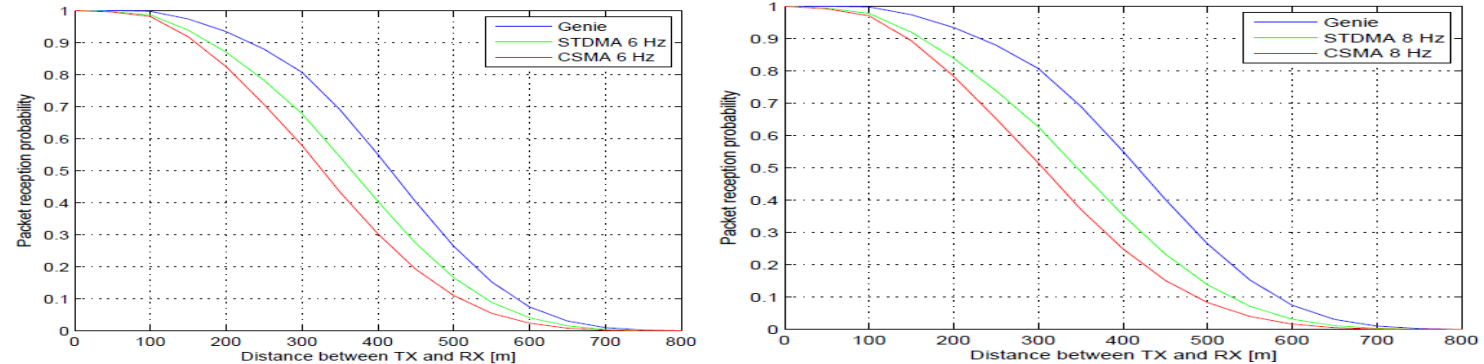

(c) Update rate $6 \mathrm{~Hz}$
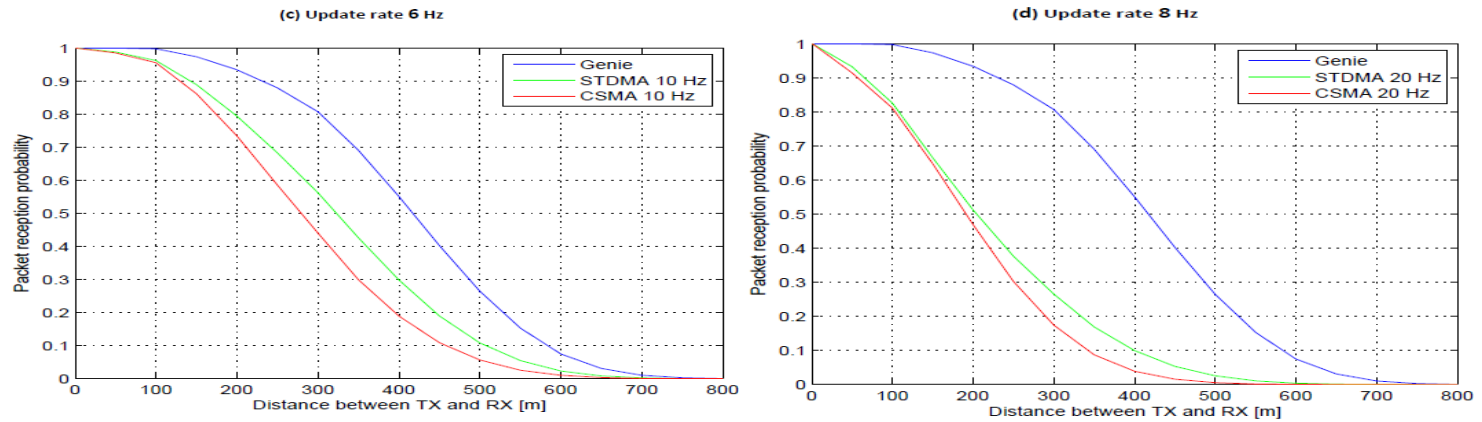

(e) Updlate rate $10 \mathrm{~Hz}$

(f) Update rate $20 \mathrm{~Hz}$

Figure 14. Packet reception probability for CSMA and STDMA when using the Nakagami model for different update rates of; (a) $2 \mathrm{~Hz}$, (b) $6 \mathrm{~Hz}$, (c) $8 \mathrm{~Hz}$, (d) $10 \mathrm{~Hz}$, (e) $16 \mathrm{~Hz}$, and (f) $20 \mathrm{~Hz}$. 


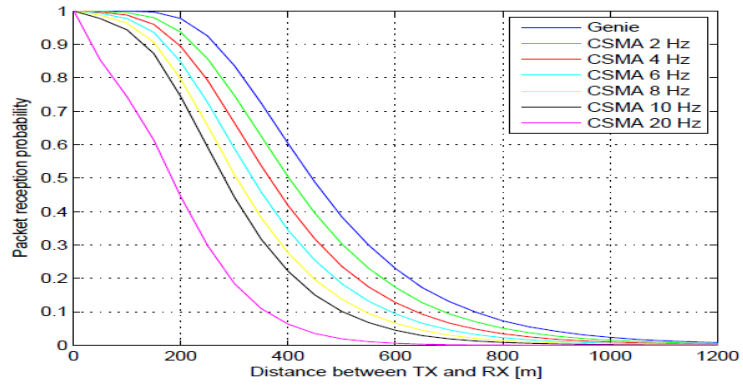

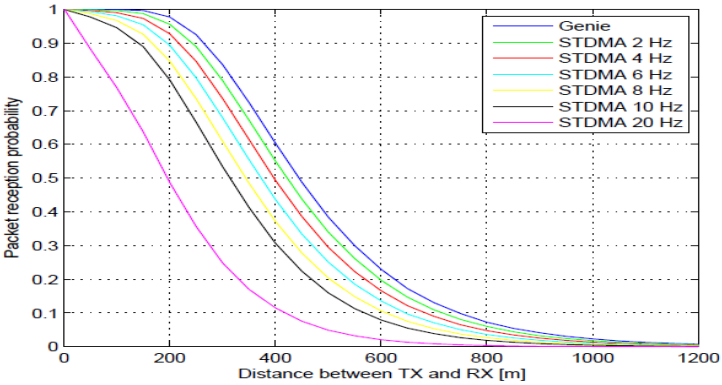

(b)

Figure 15. Packet reception probability for update rates of $2 / 4 / 6 / 8 / 10 / 20 \mathrm{~Hz}$ when using the LOS/OLOS model; (a) CSMA, and (b) STDMA

\subsection{MAC-to-MAC Delay}

MAC-to-MAC delay combines packet reception probability and channel access delay into one performance measure. The CDF for the MAC-to-MAC delay when using the Nakagami model for the update rates 2/4/6/8/10/20 Hz, is depicted on Figure 17. Since the MAC-to-MAC delay is a function of the update rate, the range of the abscissa is selected based on the specific update rate in use, i.e., the time between two packet generations (e.g., $1 /(2 \mathrm{~Hz})=0.5 \mathrm{~s})$. We use the convention that packet drops of any kind cause the MAC-to-MAC delay to be infinite. Packet drops can occur at the transmitter (for CSMA when channel access is not granted until the packet has expired) or at the receiver (for both CSMA and STDMA when decoding fails). However, on Figure 17, no packets have been dropped at the transmitting side. Therefore, the MAC-to-MAC delay is only infinite as a result of decoding failures. Every curve in the Figure represents all cases when the distance between transmitter and receiver is within a certain range, i.e., "STDMA 100-200 m" implies all receivers that are between 100-200 meters away from a transmitter.

The channel access delay for CSMA increases with increased update rate, quite in contrast to STDMA, where it instead decreases. On Figure 17(f), showing the highest update rate, the MAC-to-MAC delay reaches its maximum value after approximately the same time for both protocols for a TX-RX separation of 0-100 meters. The largest difference in performance between the MAC protocols is found on Figure 17(d) for an update rate of $8 \mathrm{~Hz}$, where CSMA shows a lower MAC-to-MAC delay for the successfully delivered packets, but where STDMA manages to deliver more packets to higher layers implying that the CDF converges to a higher value. This illustrates the basic trade-off between delay and reliability. STDMA offers better reliability than CSMA at the expense of a longer MAC-to-MAC delay. For the shortest TX-RX separation the MAC protocols perform equally well, which is consistent with the finding for the packet reception probability curves.

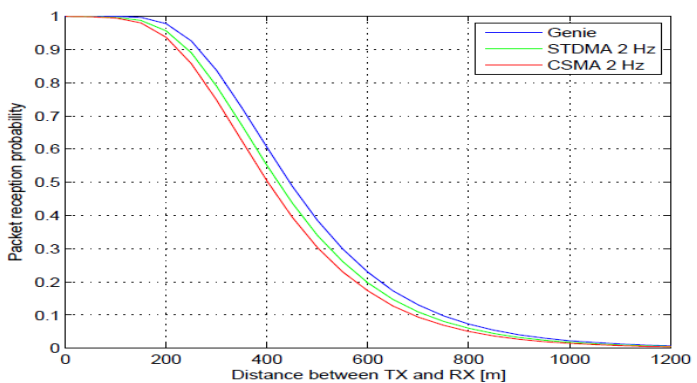

(a) Update rate $2 \mathrm{~Hz}$

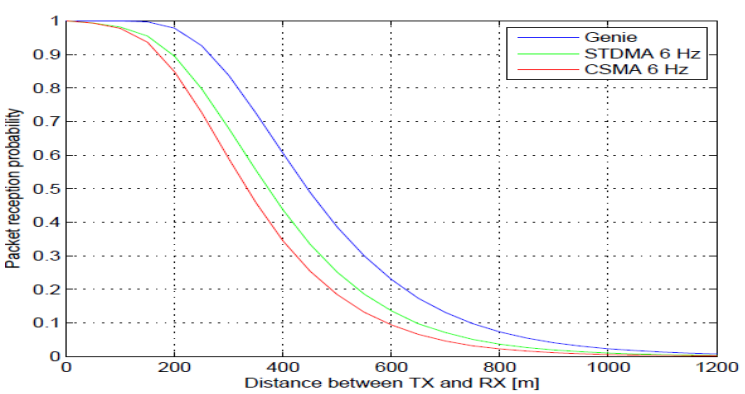

(c) Update rate $6 \mathrm{~Hz}$

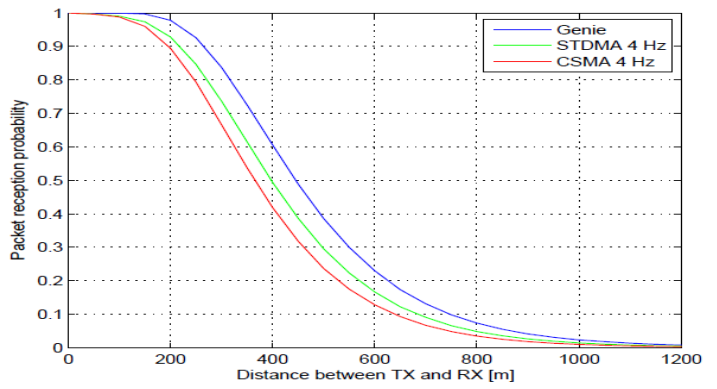

(b) Update rate $4 \mathrm{~Hz}$

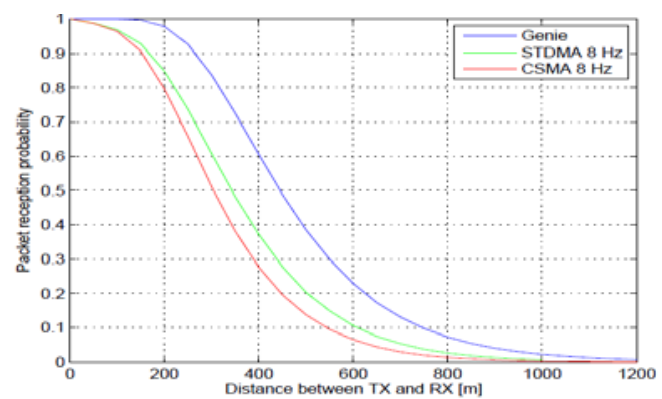

(d) Update rate $8 \mathrm{~Hz}$ 


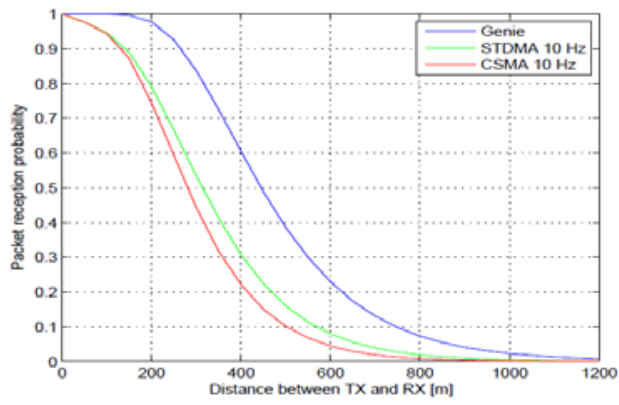

(e) Update rate $10 \mathrm{~Hz}$

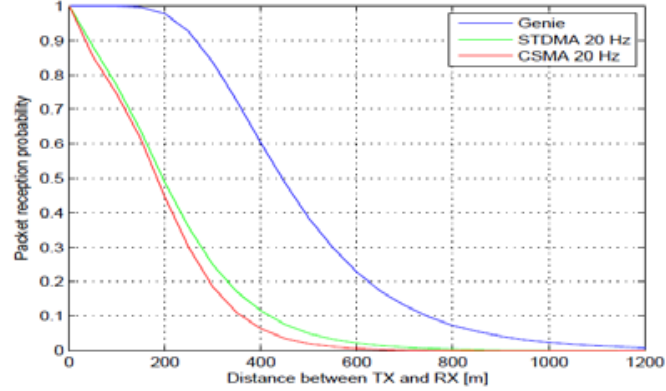

(f) Update rate $20 \mathrm{~Hz}$

Figure 16. Packet reception probability for CSMA and STDMA when using the LOS/OLOS model for different update rates of; (a) $2 \mathrm{~Hz}$, (b) $6 \mathrm{~Hz}$, (c) $8 \mathrm{~Hz}$, (d) $10 \mathrm{~Hz}$, (e) $16 \mathrm{~Hz}$, and (f) $20 \mathrm{~Hz}$.

The MAC-to-MAC delay for the LOS/OLOS model is depicted on Figure 18 for both MAC schemes. STDMA and CSMA performs equally well for a TX-RX separation less than 100 meters. For longer distances, STDMA performs better than CSMA, i.e., the delay CDF flattens out at higher value. The largest difference in performance between CSMA and STDMA is also here found for an update rate of $8 \mathrm{~Hz}$; see Figure 18(d). For every update rate, the largest difference between the two protocols is for a TX-RX separation of between 200-400 meters. We can conclude that CSMA has a lower minimum MAC-to-MAC delay, but that with STDMA, a higher percentage of all packets have a finite MAC-to-MAC delay.

\subsection{City Scenario}

The Palm-Beach Road (Nerul to Vashi) city scenario is chosen because here the highest relative speeds (i.e., $\min 60 \mathrm{~km} / \mathrm{h}$ to $\max 100$ or above $\mathrm{km} / \mathrm{h}$ ) in vehicular environments are found and hence it should constitute the biggest challenge for the MAC layer. The vehicles are entering each lane of the city road according to a Poisson process with a mean inter-arrival time of 3 seconds (consistent with the 3second-rule used in Sweden, which recommends drivers to maintain a 3 second spacing between vehicles). The speed of each vehicle is modelled as a Gaussian random variable with different mean values for each lane; $60 \mathrm{~km} / \mathrm{h}$ and $100 \mathrm{~km} / \mathrm{h}$ a standard deviation of $1 \mathrm{~m} / \mathrm{s}$. For simplicity we assume that no overtaking is possible and vehicles always remain in the same lane. There is no other data traffic in addition to the heartbeat broadcast messages. The channel model is a simple circular transmission model where all vehicles within a certain sensing range will sense and receive packets perfectly. The simulated sensing ranges are $500 \mathrm{~m}$ and $1000 \mathrm{~m}$. We have tried to focus on how the two MAC methods perform in terms of time between channel access requests until actual channel access within each vehicle node. Three different packet lengths have been considered: 500, 500 and 1000 byte. The shortest packet length is just long enough to distribute the location, direction and speed, but due to security overhead, the packets are likely longer [28]. The transfer rate is chosen to be the lowest rate supported by $802.11 \mathrm{p}$, namely 3 Mbps. Since all vehicles in the simulation are broadcasting, no ACKs are used.

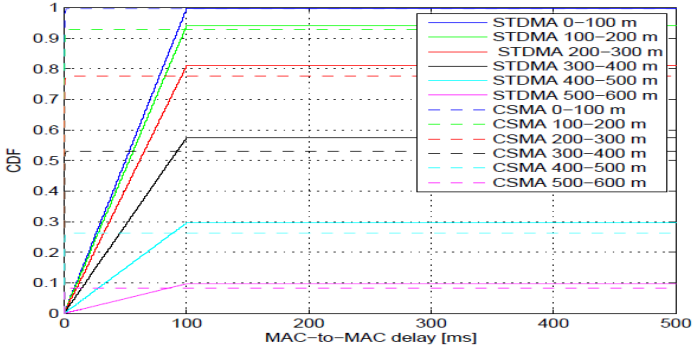

(a) Update rate $2 \mathrm{~Hz}$

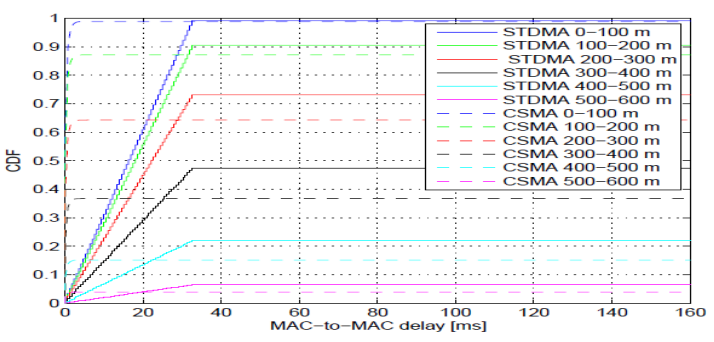

(c) Update rate 6 H

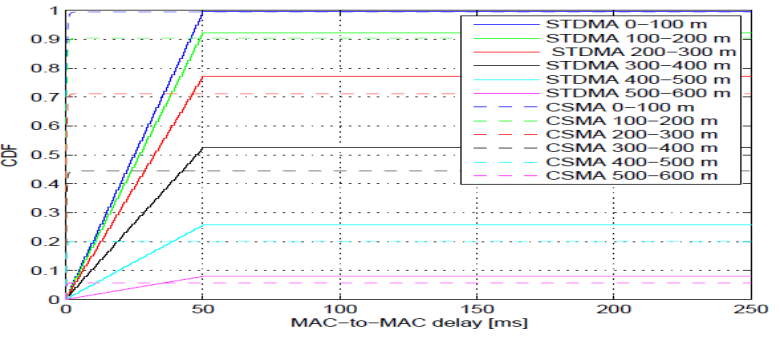

(b) Update rate $4 \mathrm{~Hz}$

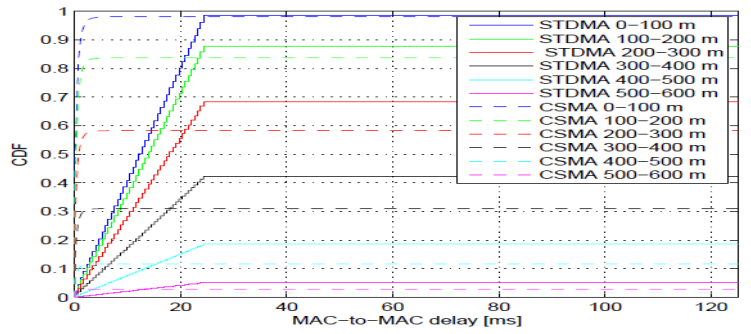

(d) Update rate $8 \mathrm{~Hz}$ 

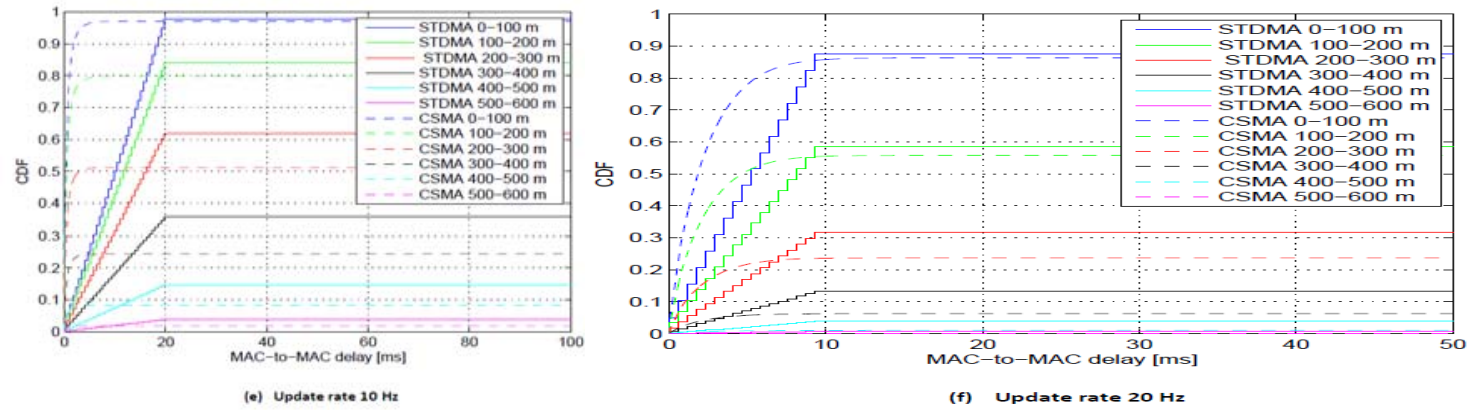

Figure 17. CDF for the MAC-to-MAC delay for CSMA and STDMA when using the Nakagami model for the following update rates: (a) $2 \mathrm{~Hz}$, (b) $4 \mathrm{~Hz}$, (c) $6 \mathrm{~Hz}$, (d) $8 \mathrm{~Hz}$, (e) $10 \mathrm{~Hz}$, and (f) $20 \mathrm{~Hz}$.

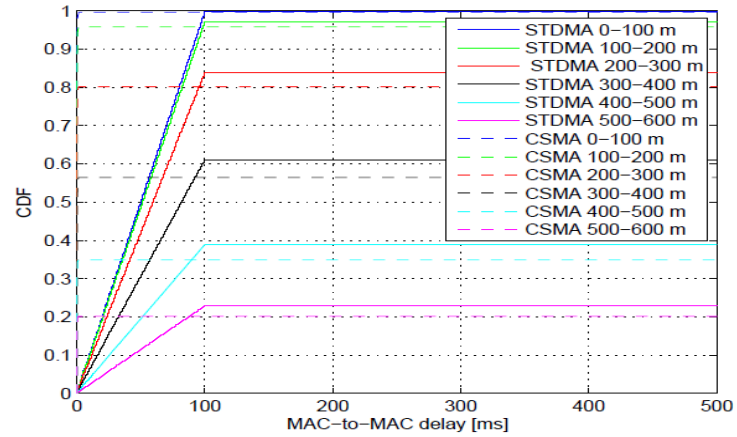

(a) Update rate $2 \mathrm{~Hz}$

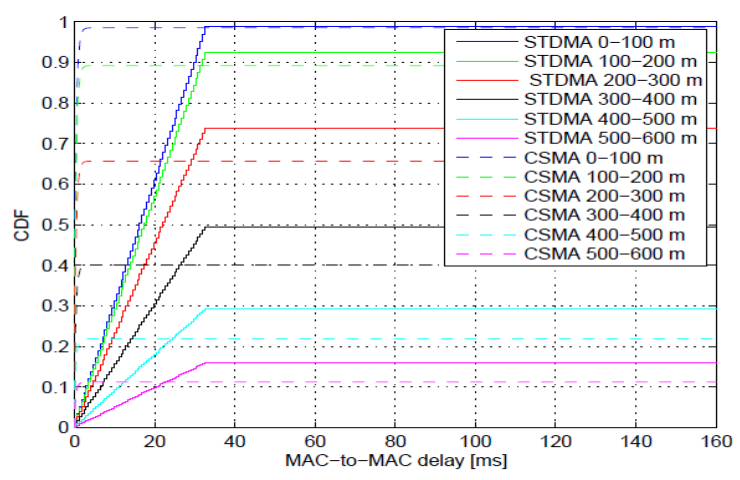

(c) Update rate $6 \mathrm{~Hz}$

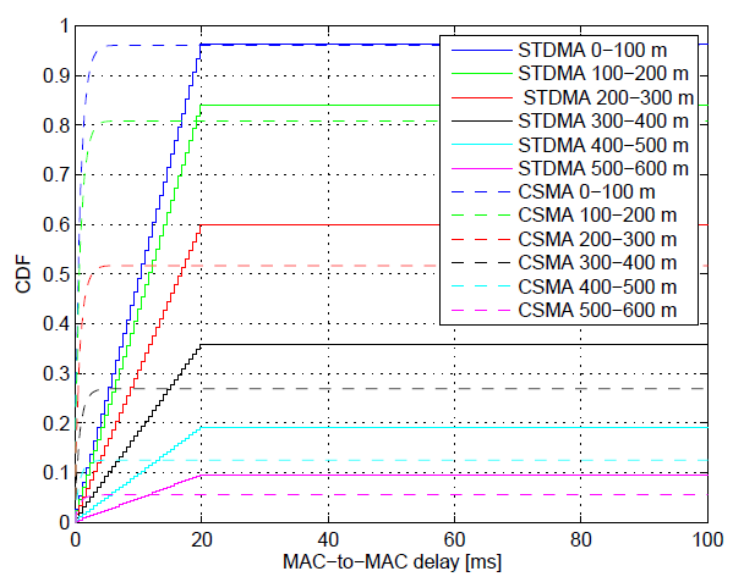

(e) Update rate $10 \mathrm{~Hz}$

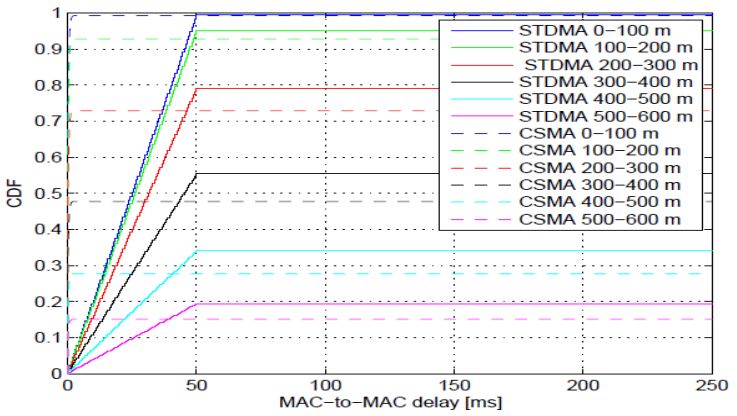

(b) Update rate $4 \mathrm{~Hz}$

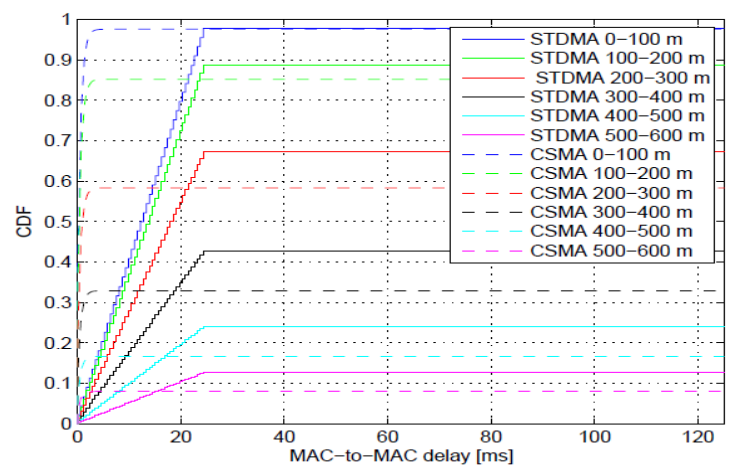

(d) Update rate $8 \mathrm{~Hz}$

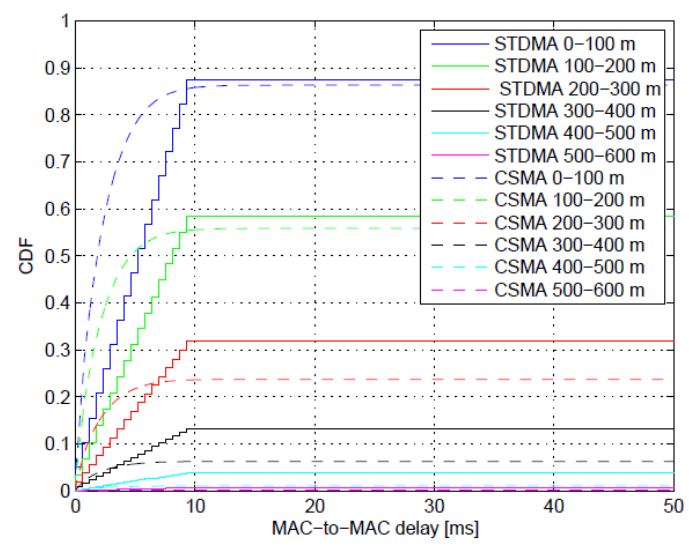

(f) Update rate $20 \mathrm{~Hz}$

Figure 18. CDF for the MAC-to-MAC delay for CSMA and STDMA when using the LOS/OLOS model for the following update rates: (a) $2 \mathrm{~Hz}$, (b) $4 \mathrm{~Hz}$, (c) $6 \mathrm{~Hz}$, (d) $8 \mathrm{~Hz}$, (e) $10 \mathrm{~Hz}$, and (f) $20 \mathrm{~Hz}$. 
On Figure 19 - the best and worst performance experienced by a single user is depicted together with the average for all users in the system. In the worst case, a vehicle node achieves successful channel access only $16 \%$ of the time i.e., $80 \%$ of all generated packets in this vehicle node are dropped. When the sensing range is 500 meters, a vehicle node will complete for the channel with approximately 230 other vehicle nodes. On Figure 20 - the results from a sensing range of $500 \mathrm{~m}$ are depicted, and the worst-case vehicle nodes are experiencing data packets drops 55\%. In this scenario, approximately 115 vehicle nodes are competing for channel access.

Table 3. Contains a summary of the simulation parameter settings for City scenario.

\begin{tabular}{|c|c|}
\hline Parameter & Value \\
\hline Start-point of Highway Road & Nerul \\
\hline Simulation Time & 30 mins. (In-time 8.30 am \& Out-time $10.00 \mathrm{am}$ ) \\
\hline Traffic direction & 2 ways \\
\hline Number of Lanes in each direction & 6 lanes ( 3 in each direction ) \\
\hline Vehicle type & Cars, Private vehicles etc. \\
\hline Speed of Vehicle nodes & $60-100 \mathrm{~km} / \mathrm{h}$ \\
\hline Communication Protocol & 802.11p and STDMA \\
\hline Traffic type & UDP \\
\hline Packet sending frequency & $5 \mathrm{~Hz}, 10 \mathrm{~Hz}$ \\
\hline Packet length & $\begin{array}{l}300 \text { bytes (Ratio } 40 \% \text { vehicles), } 500 \text { bytes (Ratio } 30 \% \text { vehicles) } \\
\text { and } 1000 \text { bytes (Ratio } 30 \% \text { vehicles). }\end{array}$ \\
\hline CWmin & 3 \\
\hline CWmax & Not used \\
\hline Communication Range & 500 meter, 1000 meter \\
\hline Back-off Time, $\mathrm{T}_{\text {Backoff }}$ & $0,9,18,27 \mu \mathrm{s}$ \\
\hline $\begin{array}{l}\text { AIFS (listening time before sending) } \\
\text { CSMA parameter }\end{array}$ & $34 \mu \mathrm{s}$ (highest priority) \\
\hline STDMA frame size & $1 \mathrm{~s}$ \\
\hline No of slots in the STDMA frame & $\begin{array}{l}1165 \text { slots ( } 300 \text { byte packets), } 718 \text { slots ( } 500 \text { byte), } 418 \text { slots } \\
\text { (1000 byte) }\end{array}$ \\
\hline
\end{tabular}

Figure $21(\mathrm{a} \& \mathrm{~b})$ - for different sensing ranges. Simulation statistics were collected from middle of the of the city scenario with the vehicle traffic. Dropped packets are considered to have infinite delays. Three plots in the figure represent $\mathrm{CDF}$ for the node performance in best average and worst case for different sensing range. In best case only $4 \%$ of generated and send packets are dropped while in worst case $60 \%$ packets are dropped for sensing range of 500 meters and $50 \%$ packets are dropped in average case for sensing range of 1000 meters. Lose of many consecutive packets, which will make the node invisible to the surrounding vehicles for a period of time. CDF for number of consecutive packet drops is on Figure 21. STDMA algorithm grants packets channel access since slots are reused if all slots are currently occupied within selection interval of the node. Node will choose the slot that is located furthest away hence there will be no packet drops at sending side when using STDMA and channel delay is small. Figure 23 - the CDF channel delay for STDMA for all nodes will choose a slot for transmission during selection interval therefore CDF for Tacc in STDMA is sending at unity after a finite delay compared to CDF for Tacc in CSMA. Figure 24 - the CDF for the minimum distance between two nodes, which utilizing the same slot within the sensing range is depicted for different packet lengths. In CSMA/CA, all channel requests did not make it to a channel access and then nodes drop packets. In CSMA/CA there is risk when nodes gets the channel access someone else also sends the packet and collision occurs. This is due to the fact that nodes can experience the channel idle at the same time, or ongoing transmission is not detected. Figure 25 - the CDF for minimum distance between two nodes in CSMA/CA city scenario sending at the same time for three different packets lengths with different ratio as shown in Table 3 above.

\section{CONCLUSIONS}

In future traffic safety system can be classified as real-time systems which mean that the data traffic sent on the wireless channel has a deadline. The most important component of a real-time vehicle- 
to-vehicle communication system is the MAC protocol method. In this paper, two MAC methods have been evaluated according to their ability to meet the real-time communication deadlines. The MAC of the vehicular communication standard IEEE 802.11p CSMA was examined through simulation, and the results indicate severe performance degradation for a heavily loaded system, both for individual nodes and for the system. The simulations show that $802.11 \mathrm{p}$ is not suitable for periodic location messages in a Mumbai-Pune Highway Road and Palm-Beach Road city scenario, if the network load is high since some nodes will drop over $85 \%$ to $90 \%$ of their data packets. Location messages will be a central part of vehicle communication systems and much traffic safety application will depend on locations. In addition, when the network load increases, the benefits of scheduling transmissions in space comes into play. With CSMA, transmissions may overlap in time, both completely and partially due to unsynchronised transmissions taking place outside the sensing range of concurrent transmitters. Also partially overlapping transmissions are likely to cause decoding failures at receivers situated in between the concurrent transmitters (the hidden terminal problem). Further, when the network load increases, CSMA stations within radio range of each other are more likely to transmit at the same time due to reaching a back-off value of zero at the same time. This occurs since CSMA stations within radio range of each other are synchronized to some extent, through the channel sensing procedure, and therefore stations tend to initiate their back-off counters at the same time, when a busy channel becomes free. The selection of back-off values is not scheduled in space and thus two or more stations can be geographically co-located when reaching a back-off value of zero, reducing the packet reception probability for many receivers.

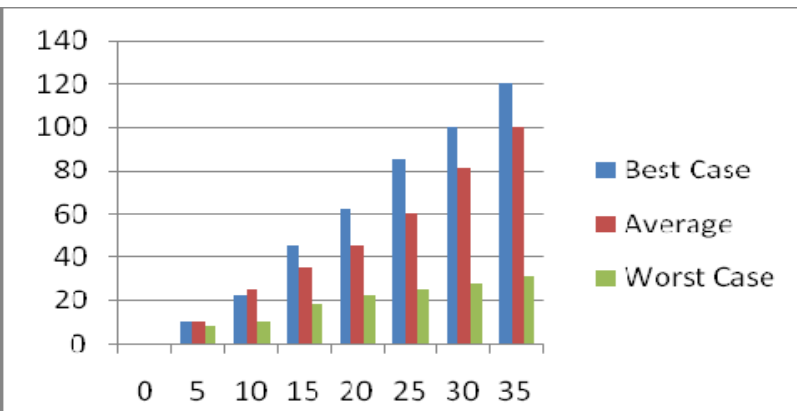

Figure 19. Channel access delay in CSMA with a sensing range of $500 \mathrm{~m}$, report rate $10 \mathrm{HZ}$ and packet length 500 byte

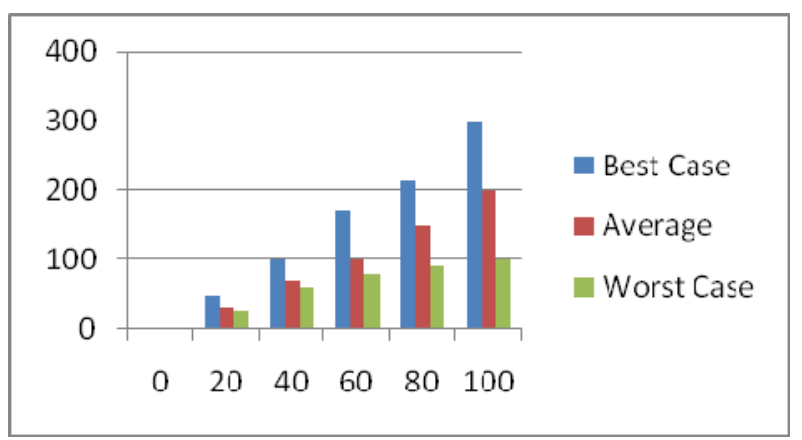

Figure 21(a). Sensing Range 500 meters

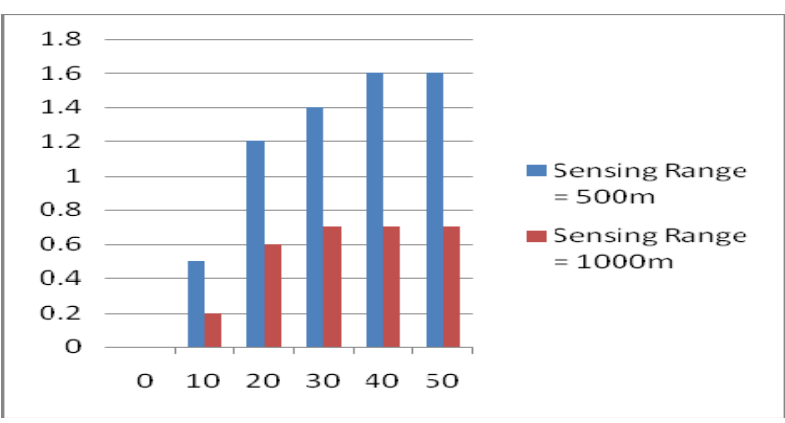

Figure 22. Number of Packets dropped due to no channel access

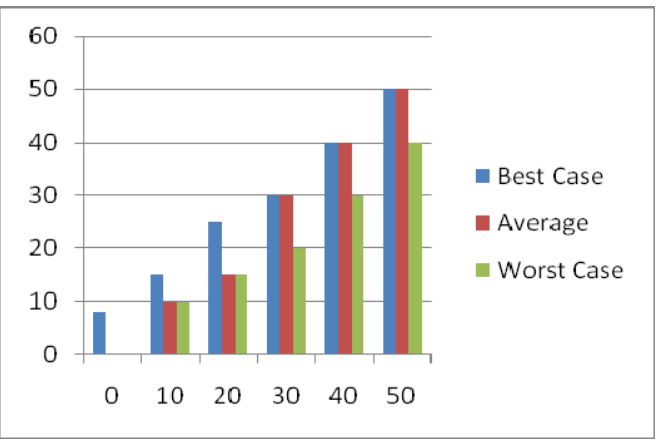

Figure 20. Channel access delay in CSMA with a sensing range of $1000 \mathrm{~m}$, report rate $10 \mathrm{HZ}$ and packet length 1000 byte

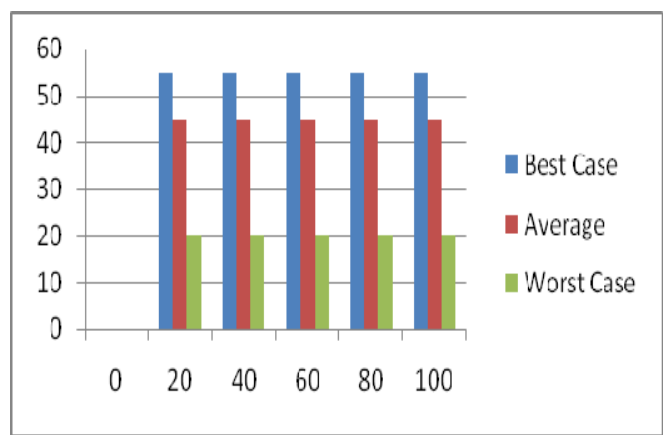

Figure 21(b). Sensing Range 1000 meters

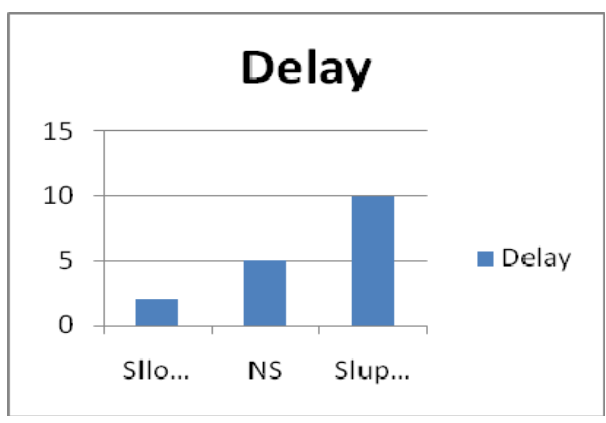

Figure 23. CDF for channel access delay in STDMA 


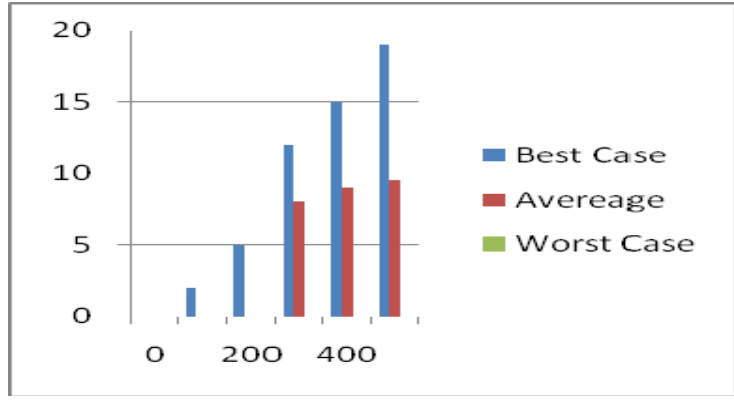

Figure 24. Utilizing the same time slot in STDMA to find minimum distance between two nodes

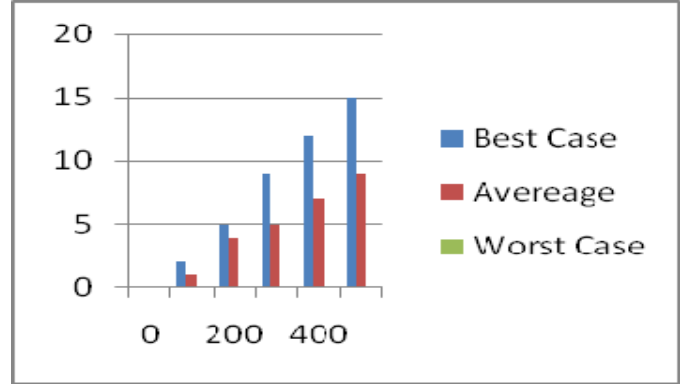

Figure 25. Sending at the same time in CSMA/CA using 300 bytes packets. $10 \mathrm{~Hz}$, sensing range $1 \mathrm{~km}$

\section{References}

1. Blum, J.J., Eskandarian, A. \& L.J. Huffman. (2004). Challenges of inter vehicle ad-hoc networks. IEEE Transactions on Intelligent Transportation System, 5(4), 347-351.

2. http://www.its.dot.gov/vii/index.htm.

3. Bilstrup, K., Uhlemann, E., Storm, E.G. \& U. Bilstrup. (2008). Evaluation of the IEEE 802.11p MAC method for vehicle-to-vehicle communication. In Proceedings of the $68^{\text {th }}$ IEEE Vehicular Technology Conference (VTC'08), September 2008 (pp.1-5). Calgary, Canada.

4. Bilstrup, K., Uhlemann, E., Storm, E.G. (2008). Medium access control in vehicular networks based on the upcoming IEEE $802.11 \mathrm{p}$ standard. In Proceedings of the $15^{\text {th }}$ World Congress on Intelligent Transport Systems (ITS'08), November 2008 (pp.1-12). New York, USA.

5. http://standards.ieee.org/board/nes/projects/802-11p.pdf

6. Bilstrup, K. (2007). A survey regarding wireless communication standards intended for a highspeed vehicle environment. Halmstad University, Sweden, Feb. 2007. (Technical Report IDE 0712).

7. Stibor, L., Zang, Y. \& H-J. Reumermann. (2007). Evaluation of communication distance of broadcast messages in a vehicular ad- hoc network using IEEE 802.11p. In Proc. IEEE Wireless Communications and Networking Conf., Mar. 2007, (pp. 254-257). Hong Kong, China.

8. Wellen, M., Westphal, B. \& P. Mähönen. (2007). Performance evaluation of IEEE 802.11-based WLANs in vehicular scenarios. In Proc. IEEE Vehicular Technology Conf., Apr. 2007 (pp. 11671171). Dublin, Ireland.

9. Xiang, W., Richardson, P. \& J. Guo. (2007). Introduction and preliminary experimental results of wireless access for vehicular environments (WAVE) systems. In Proc. Int. Conf. Mobile and Ubiquitous Systems: Network and Services, Jul. 2007 (pp. 1-8). San José, CA, US.

10. IEEE P802.11p/D3.0, Part 11: Wireless LAN Medium Access Control (MAC) and Physical Layer (PHY) Specifications: Amendment: Wireless Access in Vehicular Environments (WAVE), Draft 3.0, Jul. 2007.

11. Jiang, D. \& L. Delgrossi. (2007). IEEE 802.11: Towards an International Standard for Wireless Access in Vehicular Environments.

12. Alonso, A., Sjöberg, K., Uhlemann, E.,. Ström, E.G. \& C.F. Mecklenbräuker. (2011). Challenging Vehicular Scenarios for Self-Organizing Time Division Multiple Access. European Cooperation in the Field of Scientific and Technical Research.

13. Sjöberg Bilstrup, K., Uhlemann, E. \& E.G. Ström. (2010). Scalability issues for the MAC methods STDMA and CSMA/CA of IEEE 802.11p when used in VANETs. In Proceedings of the IEEE International Conference on Communications (ICC2010).

14. Bilstrup, K., Uhlemann, E., Ström, E.G. \& U. Bilstrup. (2009). On the Ability of the 802.11p and STDMA to provide predictable channel access. In Proceedings of the 16th World Congress on ITS.

15. IEEE Std. 802.11e-2005, Part 11: Wireless LAN Medium Access Control (MAC) and Physical Layer (PHY) Specifications: Amendment 8: Medium Access Control (MAC) Quality of Service Enhancements, 2005.

16. Bai, F. \& H. Krishnan. (2006). Reliability analysis of DSRC wireless communication for vehicle safety applications. In Proc. IEEE Intelligent Transportation Systems Conf., Sep. 2006 (pp. 355362). Toronto, Canada.

17. Blum, J., Eskandarian, A. \& L.J. Hoffman. (Dec. 2004). Challenges of inter vehicle ad hoc networks. IEEE Trans. Intelligent Transportation Systems, 5(4), 347-351.

18. Krishna, C.M. \& K.G. Shin. (1997). Real-Time Systems. New York: McGraw-Hill. 
19. Eichler, S. (2007). Performance evaluation of the IEEE 802.11p WAVE communication standard. In Proc. IEEE Vehicular Technology Conf., Oct. 2007 (pp. 2199-2203). Baltimore, MD, US.

20. Choi, N. et al. (2007). A solicitation-based IEEE 802.11p MAC protocol for roadside to vehicular networks. In Proc. Work. on Mobile Networking for Vehicular Environments, May 2007 (pp. 9196). Anchorage, AK, US,

21. Suthaputchakun, C. \& A. Ganz. (2007). Priority based inter-vehicle communication in vehicular adhoc networks using IEEE 802.11e. In Proc. IEEE Vehicular Technology Conf., Apr. 2007 (pp. 2595-2599). Dublin, Ireland.

22. Shankar, S. \& A. Yedla. (2007). MAC layer extensions for improved QoS in 802.11 based vehicular ad hoc networks. In Proc. IEEE Int. Conf. on Vehicular Electronics and Safety, Dec. 2007 (pp. 1-6). Beijing, China.

23. Sjöberg, K. (2011). Standardization of Wireless Vehicular Communications within IEEE and ETSI. IEEE VTS Workshop on Wireless Vehicular Communications.

24. Bilstrup, K., Uhlemann, E., Ström, E.G. \& U. Bilstrup. (2009). On the Ability of the 802.11p MAC Method and STDMA to Support Real-Time Vehicle-to-Vehicle Communication. EURASIP Journal on Wireless Communications and Networking, 13, 2009.

25. Alonso, A., Sjöberg, K., Uhlemann, E., Ström, E.G. \& C.F. Mecklenbräuker. (2011). Challenging Vehicular Scenarios for Self-Organizing Time Division Multiple Access. European Cooperation in the Field of Scientific and Technical Research. 